\title{
Does Three-Dimensional Printed Patient-Specific Templates Add Benefit in Revision Surgeries for Complex Pediatric Kyphoscoliosis Deformity with Sublaminar Wires in Situ? A Clinical Study
}

\author{
Kunal Shah ${ }^{1}$, Akshay Gadiya ${ }^{2}$, Munjal Shah ${ }^{3}$, Devarsh Vyas ${ }^{4}$, Priyank Patel ${ }^{5}$, Shekhar Bhojraj ${ }^{6}$, Abhay Nene \\ "We Are Spine" Centre, Aarav Polyclinic, Mumbai, India \\ ${ }^{2}$ Department of Spine Surgery, Wockhardt Hospital, Mumbai, India \\ ${ }^{3}$ Department of Orthopedic Surgery, Lilavati Hospital and Research Center, Mumbai, India \\ ${ }^{4}$ Biomedical Application Engineer, Mumbai, India \\ ${ }^{5}$ Department of Orthopedic Surgery, Jupiter Hospital, Thane, India \\ ${ }^{6}$ Department of Spine Surgery, Lilavati Hospital, Mumbai, India \\ ${ }^{7}$ Department of Spine Surgery, Wockhardt Hospital, Mumbai, India
}

Study Design: Case-control study.

Purpose: To evaluate the accuracy of three-dimensional (3D) printed patient-specific templates (PSTs) for placement of pedicle screws (PAs) in patients undergoing revision surgeries for complex kyphoscoliosis deformity with sublaminar wires in situ.

Overview of Literature: Revision kyphoscoliosis correction surgery in pediatric patients is a challenging task for the treating surgeon. In patients with sublaminar wires in situ, the native anatomical landmarks are obscured, thus making the freehand screw placement technique a highly specialized task. Hence, the concept of using PSTs for insertion of PAs in such surgeries is always intriguing and attractive.

Methods: Five consecutive patients undergoing revision deformity correction with sublaminar wires in situ were included in this study. Patients were divided in two groups based on the technique of PA insertion. A total of 91 PAs were inserted using either a freehand technique (group A) or 3D printed templates (group B) (34 vs. 57). The placement of PAs was classified according to a postoperative computed tomography scan using Neo's classification. Perforation beyond class $2(>2 \mathrm{~mm})$ was termed as a misplaced screw. The average time required for the insertion of screws was also noted.

Results: Mean age, surgical time, and blood loss were recorded. The change in mean Cobb's angle in both groups was also recorded. The difference in rates of misplaced screws was noted in group A and group B ( $36.21 \%$ vs. $2.56 \%)$; however, the mean number of misplaced PAs per patient in group $A$ and group $B$ was statistically insignificant ( $6.5 \pm 3.54$ vs. $4.67 \pm 1.53, p=0.4641)$. The mean time required to insert a single PA was also statistically insignificant ( $120 \pm 28.28$ vs. $90 \pm 30$ seconds, $p=0.3456)$.

Conclusions: Although 3D printed PSTs help to avoid the misplacement of PAs in revision deformity correction surgeries with sublaminar wires in situ, the mean number of misplaced screws per patient using this technique was found to be statistically insignificant when compared with the freehand technique in this study.

Keywords: Kyphosis; Scoliosis; Pedicle screws; Three-dimensinal printing; Patinet-specific templates

Received Jan 19, 2019; Revised Sep 19, 2019; Accepted Oct 15, 2019

Corresponding author: Akshay Gadiya

Department of Spine Surgery, Wockhardt Hospital, Anand Rao Nair Marg, Mumbai Central, Mumbai, Maharashtra 400011, India

Tel: +91-9930282040, E-mail: akshaygadia@gmail.com 


\section{Introduction}

Deformity correction surgery is regularly performed in pediatric patients, and the outcomes following these surgeries are beneficial in terms of clinical and functional outcomes [1-3]. However, the required revision deformity surgery is challenging for the patient and for the treating physician. Moreover, these surgeries are associated with complications such as wound-healing issues, respiratory complications, pseudo-arthrosis, and instrumentation failure [4-6]. Surgical challenges include poorly developed or ill-defined bony landmarks, a lack of advanced instruments and implants, and the patient's low medical capability to cope with prolonged surgeries [7]. Implant-related complications often need revision surgery, which is technically demanding and requires a high level of expertise. The incidence rate of revision spinal surgery in pediatric patients is $7.5 \%$ to $12.5 \%$ [8].

The rate of complications in revision spinal fusion in pediatric patients is higher than in primary spinal fusion (16.7\% versus $8.6 \%$ ) [9]. Most of these complications are implant-related (5.3\%) and are often concerned with the malposition of screws because of the distortion of the native anatomical landmarks for pedicle screw insertion [9]. Thus, the concept of using patient-specific and vertebraspecific templates for the insertion of pedicle screws in revision deformity correction surgeries is always intriguing and attractive. Recently, three-dimensional (3D) printing technology has been widely used for manufacturing patient-specific templates, which has been found to be useful for complex spinal deformity surgeries with successful outcomes [10,11]. Many companies in different parts of the world are commercially manufacturing these patient-specific pedicle screw templates (e.g., Firefly; Mighty Oak Medical, Englewood, CO, USA). However, their role in revision spinal surgeries, where they would be most supported, is not reported. Our study highlights the efficacy of 3D printed templates in revision pediatric kyphoscoliosis deformity surgery with previous stainlesssteel implants in situ.

\section{Materials and Methods}

This was a prospective study. Five consecutive patients (one male and four females) who had a previous spinal deformity correction using sublaminar wires and a stainless-steel spinal loop rectangle, and who subsequently underwent a revision surgery, were included in this study. All five patients underwent revision deformity correction using the pedicle screws and rod system. The etiology of the deformity was congenital in three of the patients, posttubercular in one patient, and idiopathic in one patient. The type of deformity was scoliosis in three patients, and kyphoscoliosis in the other two patients. In the first two patients, pedicle screws were inserted using the freehand technique (group A), and in the remaining three patients, pedicle screws were inserted using $3 \mathrm{D}$ printed templates (group B). Table 1 shows the epidemiological details of all patients. All patients had given their consent and institutional review board approval had been given for this study (Lilavati Hospital and Research Center, Mumbai; IRB approval no., 08/19-3 [b2]).

\section{Preoperative planning and making of templates}

Preoperatively, all patients underwent plain standing radiographs, spiral computed tomography (CT) scans of the spine with 3D reconstruction, and magnetic resonance imaging scans.

The software used to convert Digital Imaging and Communications in Medicine (DICOM) images to 3D models was the Mimics Innovation Suite (Mimics Medical and 3-Matic Medical 11; Materialise, Leuven, Belgium). The DICOM data of the patient's CT scan was imported into Mimics Medical 19 (Materialise). On preliminary inspection, it was found to be important to separate the spine from the remnant implant. This was done by first segmenting the bone and implant using appropriate threshold values. Furthermore, manual intervention in segmentation was required to eliminate the artifacts caused by the presence of the stainless-steel implant. The implant and spine were then imported into 3-Matic Medical 11 (Materialise) and were used as base data on which to design pedicle-locating guides for each vertebra, using various computer-aided design tools available in the software. A team comprising a biomedical engineer and a surgeon differentiated the metal artifacts and the bone defects so as to make templates that had the maximum potential bone contact surface. The final 3D printed model of the spine and the design of the templates are shown in Fig. 1.

\section{Surgical procedure}

Two senior spinal surgeons and the same surgical team 

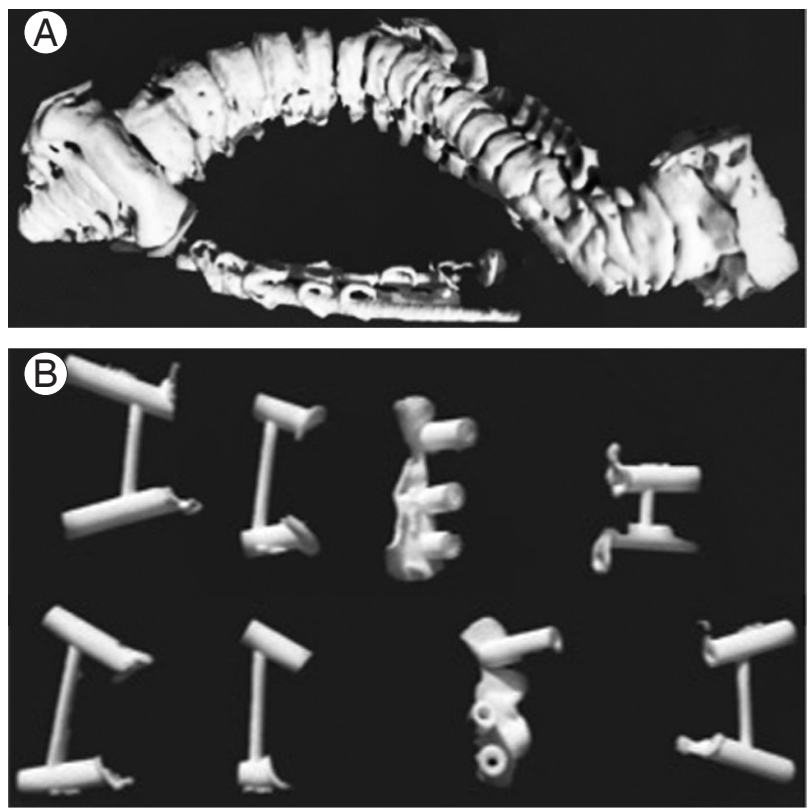

Fig. 1. (A) 3D printed biomodel of the spine and spinal rectangle loop with sublaminar wires. (B) 3D printed pedicle screw templates. 3D, three-dimensional. performed all the surgeries. All patients underwent a single-stage removal of the sublaminar wires and spinal rectangle and revision deformity correction using the standard titanium pedicle screws and rod system. Spinal exposure was achieved taking care to remove all soft tissue and to preserve bone, such that the templates would fit snuggly.

The removal of the sublaminar wires/spinal loop rectangle was done carefully, so bony landmarks were not affected. The 3D printed templates were then used for inserting the pedicle screws in group B patients. Spinal cord monitoring was used in all patients during surgery. No patient had intraoperative signal changes, either motor evoked potentials or somatosensory evoked potentials, during surgery. No patient had neurological worsening postoperatively (Fig. 2).
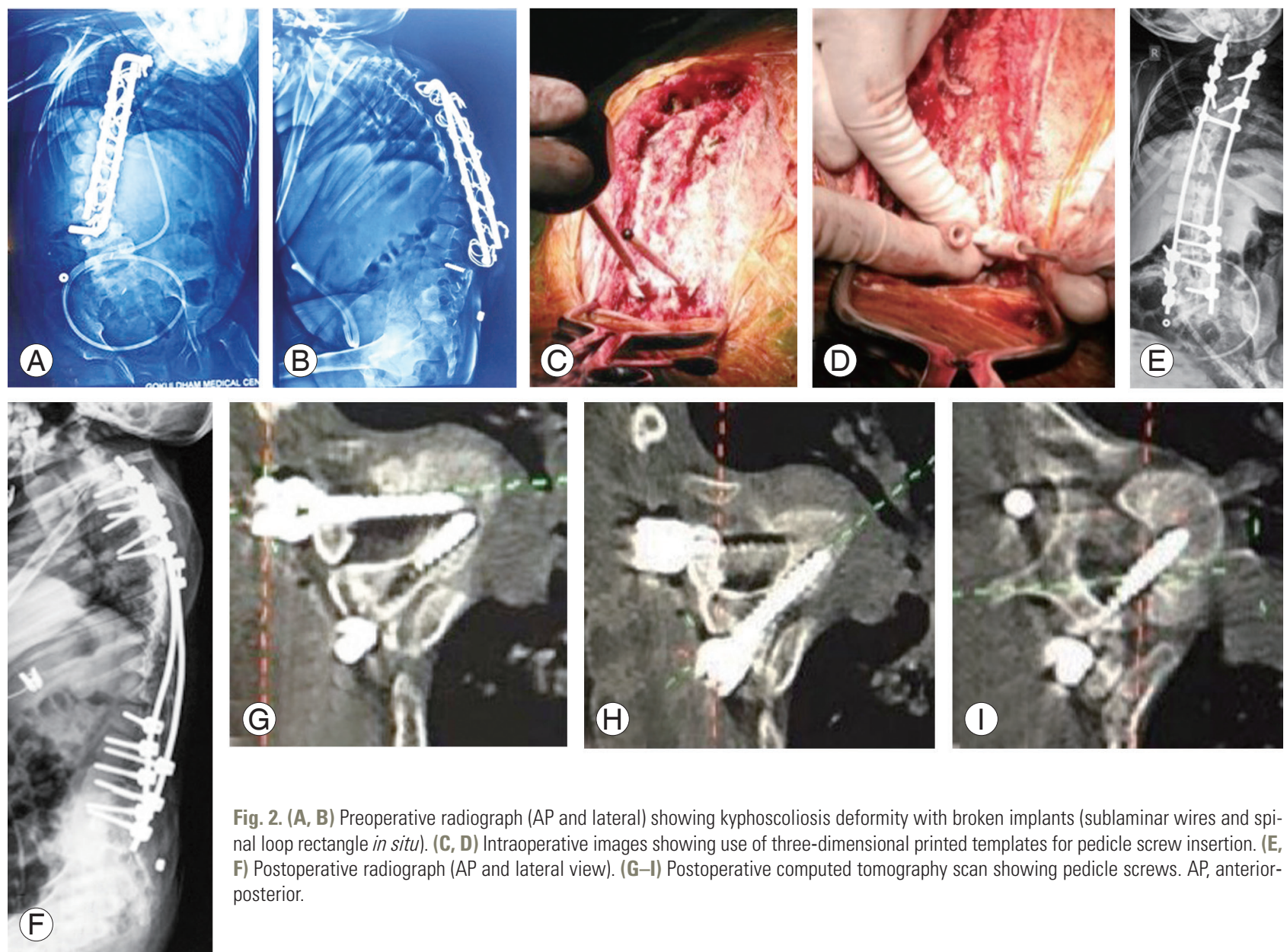

Fig. 2. (A, B) Preoperative radiograph (AP and lateral) showing kyphoscoliosis deformity with broken implants (sublaminar wires and spinal loop rectangle in situ). (C, D) Intraoperative images showing use of three-dimensional printed templates for pedicle screw insertion. ( $\mathbf{E}_{\text {s }}$ F) Postoperative radiograph (AP and lateral view). (G-I) Postoperative computed tomography scan showing pedicle screws. AP, anteriorposterior. 


\section{Postoperative course}

Following surgery patients were mobilized out of bed depending on their pain tolerance. No brace support was used. The CT scans were performed in the postoperative period at first follow-up (at 3 months) in all patients to study the placement of the pedicle screws. A screw was designated as a misplaced screw if it was found to be perforating the respective pedicle (a breach in either the superior, inferior, medial, or lateral cortex) beyond $2 \mathrm{~mm}$ as described by Neo et al. [12].

\section{Results}

The mean age of the patients in the study group was $12.2 \pm 2.2$ years. The mean surgical time was $238 \pm 57.62$ minutes. The mean blood loss was $610 \pm 277.04 \mathrm{~mL}$. Of the total 91 pedicle screws, 34 pedicle screws were inserted using the freehand technique, and 57 pedicle screws were inserted using $3 \mathrm{D}$ printed templates. The mean preoperative Cobb's angle in group A was $54^{\circ} \pm 19.8^{\circ}$, while that in group $\mathrm{B}$ was $79.33^{\circ} \pm 13.61^{\circ}$. The mean postoperative Cobb's angle in group A was $27^{\circ} \pm 4.4^{\circ}$, while that in group $B$ was $31.33^{\circ} \pm 10.26^{\circ}$. The total number of misplaced pedicle screws in group A was 13, while that in group B was 14 (Table 1). The rate of misplaced screws in group A was $36.21 \%$, while that in group B was $24.56 \%$ (Table 1). The mean number of misplaced pedicle screws per patient in group A was $6.5 \pm 3.54$, while that in group B was $4.67 \pm 1.53$; this was statistically insignificant $(p=0.4641)$. The mean time required to insert a single pedicle screw in group $A$ was $120 \pm 28.28$ seconds, while that in group B was $90 \pm 30$ seconds; this was statistically insignificant $(p=0.345)$ (Table 2).

\section{Discussion}

Traditionally, pedicle screws are inserted in the lumbar spine using a freehand technique; however, pedicle screw insertion in the thoracic spine is more challenging due to the complex native anatomy $[13,14]$. Insertion of pedicle screws by this method is based on preoperative radiology, the intraoperative native bony anatomy, and the tactile feedback of the surgeon. Placement of pedicle screws in kyphoscoliosis correction surgery requires technical expertise owing to the vertebral rotation and associated congenital bony malformations. Rigid deformities are

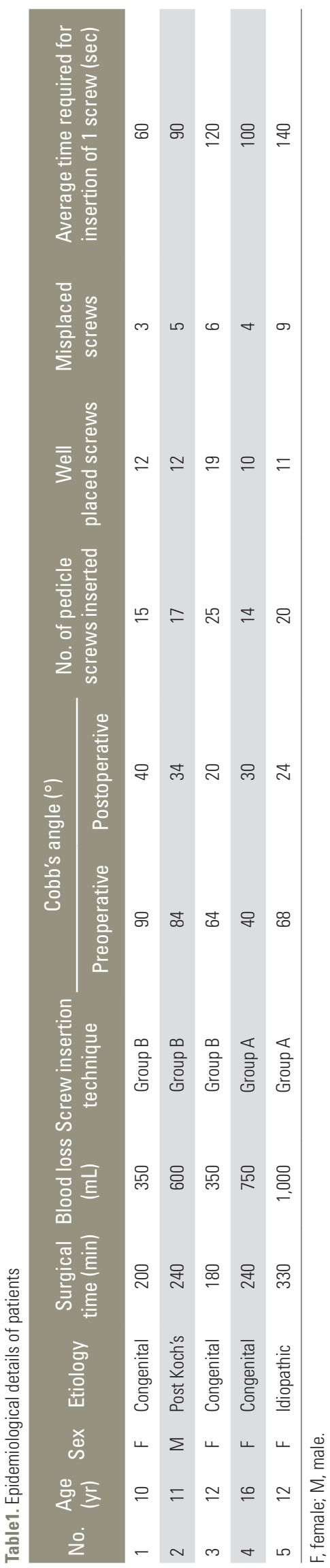


Table 2. Details of pedicle screws in both groups

\begin{tabular}{lccc} 
Variable & Group A & Group B & $p$-value \\
Average time required to insert 1 screw (sec) & $120 \pm 28.28$ & $90 \pm 30$ & $0.3456^{\text {a) }}$ \\
No. of misplaced screws & $6.50 \pm 3.54$ & $4.67 \pm 1.53$ & $0.4641^{\text {b) }}$ \\
\hline No. of pedicle screws inserted & $17 \pm 4.24$ & $19 \pm 5.29$ & $0.6889^{\text {b) }}$ \\
\hline
\end{tabular}

Values are presented as mean \pm standard deviation.

${ }^{\text {al }}$ Calculated using $t$-test. ${ }^{\text {b) } C a l c u l a t e d ~ u s i n g ~ c h i-s q u a r e ~ t e s t . ~}$

often associated with anomalies of the thoracic spine pedicle [15]. The risk of injury to the spinal cord increases on the concave side of the deformity because the pedicles are thinner, sclerotic, and dysplastic and the spinal cord lies in direct contact with the medial wall of the concave pedicles. The rate of screw malposition in scoliosis surgery is reported as being $4.2 \%$ to $15 \%$ [16]. These problems are multiplied in the pediatric population due to difficulties in instrumentation, growth of the vertebral column, and lung development.

To address this problem, various techniques of safe pedicle screw placement have been described in the literature, including intraoperative fluoroscopy, computer-assisted navigation based on the preoperative CT scan, O-armbased navigation, robotic-assisted pedicle screw placement, use of a PediGuard, and use of 3D printed templates [17-22].

Various authors have previously reported on use of navigation based on preoperative CT scans for safe placement of pedicle screws in deformity correction of the spine $[23,24]$. The pedicle screw misplacement with this technique is reported as being between $1.8 \%$ and $11.4 \%$ $[23,24]$. In patients undergoing revision deformity correction with sublaminar wires and rectangle loop in situ, there are many metal artifacts causing differences in anatomical relationships, as determined by the preoperative CT scan and intraoperative findings. Also, selecting starting points for pedicle screw placement on $3 \mathrm{D}$ reconstruction of the preoperative CT scan is not possible in these patients.

$\mathrm{O}$-arm-based navigation has an advantage over the previous technique as it provides an intraoperative CT scan of the patient for safe placement of the pedicle screws. The operating surgeon does not need to rely on the preoperative CT scan, which may be obscured with metal artifacts that make the bony landmarks difficult to identify. However, intraoperative CT scans are associated with increased radiation exposure, both to the surgical team and the patient [25]. Moreover, the cost of equipment and space occupied by the $\mathrm{O}$-arm is relatively high when compared with CT-based navigation. The rate of pedicle screw misplacement in scoliosis surgery using $\mathrm{O}$-arm navigation has been reported to be approximately $3.1 \%$ [18].

Recently, many studies have reported the use of robotics in spinal surgery for safe placement of pedicle screws $[19,26,27]$. However, their use in scoliosis surgery is scarcely described in the literature [19,27]. In adolescent idiopathic scoliosis, the rate of misplacement in pedicle screws with robotic-assisted placement has been reported to be $7.2 \%$ [19]. However, the use of robotics for placement of pedicle screws cannot be universalized due to the cost constraints, especially in developing countries, and the complexity and time involved in registering the data and operating it, and an associated steep learning curve. Also, the space occupied by the robot in the operating room is a concern.

With the advancement in technology, the role of 3D printing in preoperative planning and execution of complex spinal surgery has been increasingly explored in the last two decades [28]. Application of 3D printing (additive manufacturing) to manufacture the custom-made templates for insertion of the pedicle screws in complex spinal surgeries is well reported in the literature [29,30]. Using patient-specific data to create templates that can be used during surgery reduces the risk associated with misplaced pedicle screws. The 3D printed templates provide simple, convenient, low cost, and complex-equipment-free alternatives to increase the accuracy of pedicle screw placement in complex spinal surgeries [29]. The advantages can also be realized for insertion of screws in patients undergoing revision surgery for deformity correction who have sublaminar wires and a stainless-steel rectangle loop in situ.

Takemoto et al. [31] evaluated the efficacy of placement of a thoracic pedicle screw using patient-specific templates made by $3 \mathrm{D}$ printing technology. Out of 40 patients, 
36 had scoliosis as the primary indication of surgery. The authors reported a $98.4 \%$ success rate for placement of pedicle screws using the patient-specific templates in scoliosis surgery. However, none of the patients in the study group had revision deformity correction.

Liu et al. [32] compared the freehand technique of pedicle screw insertion with the technique utilizing the $3 \mathrm{D}$ printed templates in ten patients who were undergoing deformity correction surgery for severe rigid scoliosis. The success rate was $93.8 \%$ for pedicle screw placement using $3 \mathrm{D}$ printed drill guides, while it was $78.8 \%$ for pedicle screw insertion using the freehand technique. No screw-related complications were reported. However, in this study group, none of the patients were operated on previously for deformity correction.

The rate of misplaced pedicle screws in our study using the freehand technique was $36.21 \%$, which was very similar to the rate described in previous studies. The rate of misplacement of pedicle screws using 3D printed templates in our study group was $24.56 \%$. The higher rate of pedicle screw misplacement in the present study can be attributed to the metal artifacts/bone defects causing difficulty in making base plates for the $3 \mathrm{D}$ printed templates. This cohort poses special challenges for making 3D printed templates and requires a skilled biomedical engineer and an experienced surgeon to manually segregate the metal artifacts and previous bone defects based on the preoperative CT scan and to make templates with maximum contact of the base plate with the remnant bone.

Our study had few limitations. It had a small sample size; however, this can be attributed to revision of sublaminar wires with pedicle screws being a rare scenario. We could not compare apical screws, or screws near previous bone defects, with relatively easier lumbar screws, or screws near virgin spine, due to the small sample size. We believe that $3 \mathrm{D}$ printed templates are a useful tool in the pediatric spinal deformity surgeon's armory for safe pedicle screw placement in primary complex deformity surgeries. Its potential in revision deformity surgeries with previous stainless-steel implants in situ seems like a possible exciting advance.

\section{Conclusions}

Pedicle screw fixation in revision surgeries for complex pediatric kyphoscoliosis deformity (especially with sublaminar wires in situ) poses a substantial challenge for the operating surgeon. The rate of misplaced pedicle screws (in this subset of the population) in the present study with the freehand technique is clinically higher than that compared with use of 3D printed patient-specific pedicle screw templates; however, this was not statistically significant. This novel technique of using 3D printed pedicle screw templates has advantages in revision surgeries of complex pediatric kyphoscoliosis deformity. With further improvement in manufacturing technology of these templates, the rate of pedicle screw misplacement will definitely reduce further in this particular subset of patients.

\section{Conflict of Interest}

No potential conflict of interest relevant to this article was reported.

\section{References}

1. Fu KM, Smith JS, Polly DW, et al. Morbidity and mortality associated with spinal surgery in children: a review of the Scoliosis Research Society morbidity and mortality database. J Neurosurg Pediatr 2011;7:37-41.

2. Helenius I, Remes V, Lamberg T, Schlenzka D, Poussa M. Long-term health-related quality of life after surgery for adolescent idiopathic scoliosis and spondylolisthesis. J Bone Joint Surg Am 2008;90:1231-9.

3. Kepler CK, Meredith DS, Green DW, Widmann RF. Long-term outcomes after posterior spine fusion for adolescent idiopathic scoliosis. Curr Opin Pediatr 2012;24:68-75.

4. Dede O, Yazici M. Revision surgery for pediatric spine deformity: corrective osteotomies. J Pediatr Orthop 2014;34 Suppl 1:S6-10.

5. Kim HJ, Cunningham ME, Boachie-Adjei O. Revision spine surgery to manage pediatric deformity. J Am Acad Orthop Surg 2010;18:739-48.

6. Yagi M, King AB, Kim HJ, Cunningham ME, Boachie-Adjei O. Outcome of revision surgery in pediatric spine deformity patients. Spine Deform 2013;1:59-67.

7. Ramo BA, Richards BS. Repeat surgical interventions following "definitive" instrumentation and fusion for idiopathic scoliosis: five-year update on a previously published cohort. Spine (Phila Pa 1976) 2012;37:1211-7. 
8. Richards BS, Hasley BP, Casey VF. Repeat surgical interventions following "definitive" instrumentation and fusion for idiopathic scoliosis. Spine (Phila Pa 1976) 2006;31:3018-26.

9. De la Garza Ramos R, Goodwin CR, Purvis T, Karikari IO, Samdani AF, Sciubba DM. Primary versus revision spinal fusion in children: an analysis of 74,525 cases from the nationwide inpatient sample. Spine (Phila Pa 1976) 2017;42:E660-5.

10. Mao K, Wang Y, Xiao S, et al. Clinical application of computer-designed polystyrene models in complex severe spinal deformities: a pilot study. Eur Spine J 2010;19:797-802.

11. Wu ZX, Huang LY, Sang HX, et al. Accuracy and safety assessment of pedicle screw placement using the rapid prototyping technique in severe congenital scoliosis. J Spinal Disord Tech 2011;24:444-50.

12. Neo M, Sakamoto T, Fujibayashi S, Nakamura T. The clinical risk of vertebral artery injury from cervical pedicle screws inserted in degenerative vertebrae. Spine (Phila Pa 1976) 2005;30:2800-5.

13. Motiei-Langroudi R, Sadeghian H. Assessment of pedicle screw placement accuracy in thoracolumbosacral spine using freehand technique aided by lateral fluoroscopy: results of postoperative computed tomography in 114 patients. Spine J 2015;15:700-4.

14. Gelalis ID, Paschos NK, Pakos EE, et al. Accuracy of pedicle screw placement: a systematic review of prospective in vivo studies comparing free hand, fluoroscopy guidance and navigation techniques. Eur Spine J 2012;21:247-55.

15. Liljenqvist UR, Halm HF, Link TM. Pedicle screw instrumentation of the thoracic spine in idiopathic scoliosis. Spine (Phila Pa 1976) 1997;22:2239-45.

16. Hicks JM, Singla A, Shen FH, Arlet V. Complications of pedicle screw fixation in scoliosis surgery: a systematic review. Spine (Phila Pa 1976) 2010;35:E46570.

17. Merloz P, Troccaz J, Vouaillat H, et al. Fluoroscopybased navigation system in spine surgery. Proc Inst Mech Eng H 2007;221:813-20.

18. Kotani T, Akazawa T, Sakuma T, et al. Accuracy of pedicle screw placement in scoliosis surgery: a comparison between conventional computed tomography-based and $\mathrm{O}$-arm-based navigation techniques. Asian Spine J 2014;8:331-8.

19. Macke JJ, Woo R, Varich L. Accuracy of robot-assist- ed pedicle screw placement for adolescent idiopathic scoliosis in the pediatric population. J Robot Surg 2016;10:145-50.

20. Chaput CD, George K, Samdani AF, Williams JI, Gaughan J, Betz RR. Reduction in radiation (fluoroscopy) while maintaining safe placement of pedicle screws during lumbar spine fusion. Spine (Phila $\mathrm{Pa}$ 1976) 2012;37:E1305-9.

21. Chen H, Guo K, Yang H, Wu D, Yuan F. Thoracic pedicle screw placement guide plate produced by three-dimensional (3-D) laser printing. Med Sci Monit 2016;22:1682-6.

22. Gadia A, Shah K, Nene A. Emergence of three-dimensional printing technology and its utility in spine surgery. Asian Spine J 2018;12:365-71.

23. Kotani Y, Abumi K, Ito M, et al. Accuracy analysis of pedicle screw placement in posterior scoliosis surgery: comparison between conventional fluoroscopic and computer-assisted technique. Spine (Phila Pa 1976) 2007;32:1543-50.

24. Sakai Y, Matsuyama Y, Nakamura H, et al. Segmental pedicle screwing for idiopathic scoliosis using computer-assisted surgery. J Spinal Disord Tech 2008;21:181-6.

25. Pitteloud N, Gamulin A, Barea C, Damet J, Racloz G, Sans-Merce M. Radiation exposure using the $\mathrm{O}-\operatorname{arm}(\mathrm{R})$ surgical imaging system. Eur Spine J 2017;26:651-7.

26. Barzilay Y, Liebergall M, Fridlander A, Knoller N. Miniature robotic guidance for spine surgery: introduction of a novel system and analysis of challenges encountered during the clinical development phase at two spine centres. Int J Med Robot 2006;2:146-53.

27. Laudato PA, Pierzchala K, Schizas C. Pedicle screw insertion accuracy using $\mathrm{O}$-arm, robotic guidance, or freehand technique: a comparative study. Spine (Phila Pa 1976) 2018;43:E373-8.

28. D'Urso PS, Williamson OD, Thompson RG. Biomodeling as an aid to spinal instrumentation. Spine (Phila Pa 1976) 2005;30:2841-5.

29. Guo F, Dai J, Zhang J, et al. Individualized 3D printing navigation template for pedicle screw fixation in upper cervical spine. PLoS One 2017;12:e0171509.

30. Sugawara T, Higashiyama N, Kaneyama S, Sumi M. Accurate and simple screw insertion procedure with patient-specific screw guide templates for posterior C1-C2 fixation. Spine (Phila Pa 1976) 2017;42:E340-6. 
31. Takemoto M, Fujibayashi S, Ota E, et al. Additivemanufactured patient-specific titanium templates for thoracic pedicle screw placement: novel design with reduced contact area. Eur Spine J 2016;25:1698-705.
32. Liu K, Zhang Q, Li X, et al. Preliminary application of a multi-level 3D printing drill guide template for pedicle screw placement in severe and rigid scoliosis. Eur Spine J 2017;26:1684-9. 